\title{
Reducing Energy Consumption in Pharmaceutical Production Processes: Framework and Case Study
}

\author{
Georg Müller • Hirokazu Sugiyama • Simon Stocker • \\ Rainer Schmidt
}

Published online: 26 July 2014

(C) Springer Science+Business Media New York 2014

\begin{abstract}
Purpose This paper aims to present a novel framework for reducing energy consumption in pharmaceutical manufacturing processes, with a primary focus on parenterals production. The framework supports (a) the design of optimal energysaving solutions, (b) the execution and implementation and (c) the comparison of designed and actual performance as a postimplementation control. In selecting promising options, multiobjective criteria are defined for comprehensive decision-making which considers not only energy savings, but also other aspects such as good manufacturing practice (GMP), risk, and workers' safety.

Methods and Framework In the framework, five phases were defined in total, with three for design and two for execution and control, respectively. In the three design phases, options are generated, evaluated and selected step by step, with appropriate
\end{abstract}

\section{G. Müller}

Institute for Chemical and Bioengineering, ETH Zurich,

Vladimir-Prelog-Weg 1-5, 8093 Zurich, Switzerland

G. Müller $(\bowtie) \cdot S$. Stocker

Site Engineering Kaiseraugst, F. Hoffmann-La Roche Ltd., Grenzacherstrasse 124, 4070 Basel, Switzerland

e-mail: georg.mueller.gm2@roche.com

S. Stocker

e-mail: simon.stocker@roche.com

H. Sugiyama $\cdot$ R. Schmidt

Pharma Technical Operations Biologics, F. Hoffmann-La Roche

Ltd., Grenzacherstrasse 124, 4070 Basel, Switzerland

H. Sugiyama

e-mail: sugiyama@chemsys.t.u-tokyo.ac.jp

R. Schmidt

e-mail: rainer.schmidt@roche.com

H. Sugiyama

Department of Chemical System Engineering, The University of

Tokyo, 7-3-1, Hongo, Bunkyo-ku, 113-8657 Tokyo, Japan evaluation criteria covering financial as well as non-financial aspects. The roles of various stakeholders, e.g., Operations, Engineering, or Quality Assurance, are defined for each phase in order to enable smooth and certain decision-making.

Case Study and Results A case study was performed in the parenterals production plant at Hoffmann-La Roche in Kaiseraugst. Twelve energy efficiency ideas were generated and, after the screening and selection process, the three most promising options from the multiobjective Pareto optimization were implemented to reduce the total plant's energy consumption by $2.5 \%$.

Conclusions The framework enabled the systematic generation and selection of various options, which helped in the allocation of company resources in a prioritized and thus effective manner.

Keywords Energy efficiency · Pharmaceutical manufacturing $\cdot$ Multiobjective decision-making . Parenterals production $\cdot$ Industrial case study

\section{Introduction}

Energy efficiency is becoming more and more important in the pharmaceutical industry as energy prices and supply uncertainty increase. Especially for operations in countries such as Switzerland, primary resources have to be imported which are susceptible to political crises and natural disasters. As a consequence of the Fukushima Daiichi nuclear disaster, several countries decided to phase out nuclear power. For example, in 2010 , nuclear power accounted for $38 \%$ of the electricity in Switzerland [1] and will have to be substituted by other sources in the upcoming decades. This restructuring of the energy sector is expected to lead to greater uncertainty on the energy market and consequently to higher prices. Under these conditions, energy conservation is being promoted which has, in addition to environmental benefits, other non-neglectable benefits, such 
as better workplace conditions, productivity, safety, shareholder value, and public relations [2]. Thus, the effects of these conditions as part of a company's sustainability strategy influence shareholders and have been recently quantified, e.g., in the Dow Jones Sustainability Index [3].

To improve a company's energy efficiency, the various sites, buildings - including plants as well as office and laboratory buildings - have to be integrated in the company's strategy. In plants/chemical manufacturing facilities, the necessary energy savings can be achieved either by means of energy efficient design or by analyzing and retrofitting existing plants/facilities. Methods and frameworks for energy conservation are practically tradition in the chemical bulk industry. Recently developed methods for structured approaches with different stages for the design and evaluation under environmentally friendly conditions are described in chemical process design and development by Sugiyama et al. [4] and Chen et al. [5]. Hoffmann et al. investigated decision-making under uncertainty [6] and uses the concept of Pareto optimization for determining the most promising process options [7]. These methods were mainly developed for commodity chemicals and continuous processes, while batch processes are used for specialty chemicals and the pharmaceutical industry. Albrecht et al. [8] incorporates this characteristic and proposes a design framework of environmentally friendly batch processes as an extension of the work of Sugiyama et al. [4]. In their joint work, Heinzle et al. and Koller et al. describe a procedure for ecological and economic assessment during the process design stage for fine chemicals and active pharmaceutical ingredient (API) production in [9, 10]. A method for assessing integral resource consumption in a multipurpose API production plant is shown in van der Vorst et al. [11]. With the aim of waste minimization, Raymond applied life cycle assessment (LCA) to solvent used in API production, and revealed the importance of solvent recovery regarding cradle-to-grave environmental impacts [12]. Furthermore, various authors have described investigations to improve the energy efficiency of parts of buildings and various unit operations, i.e., individual energy-saving solutions. With the aim of energy optimization, modeling methods for multipurpose batch plants have been published by Bieler et al. [13, 14] and Szijjarto et al. [15]. Galitsky et al. describe for the US Environmental Protection Agency (EPA) [2] a set of individual methods to improve energy efficiency in pharmaceutical buildings, ranging from offices to production facilities. Shamkishore et al. delineate different opportunities for energy efficiency in pharmaceutical manufacturing, e.g., for light, motors, and steam traps [16]. Liu [17] assesses the energy flows of an office and a pharmaceutical production building and proposes advantageous options to reduce energy consumption, in particular for heating and HVAC (heating, ventilation and air conditioning) systems. Graf highlights various strategies for the energy efficient production of pharmaceutical water [18]. Regarding energy audit, which is becoming popular to improve energy efficiency in a company, Zhi-dong et al. [19] proposes a procedure and key factors to be considered for the pharmaceutical manufacturers. In summary, various tools and recommendations have become available for designing and retrofitting chemical and pharmaceutical production processes which consider energy consumption. However, in order to turn these theories into practice, companies still need a business model/standardized procedure, that defines how to execute these design/re-design methods with consideration on good manufacturing practice (GMP), in addition to energy reduction.

In this work, a framework for energy optimization in the pharmaceutical industry was developed with a focus on parenterals manufacturing. It supports (a) the design and evaluation of optimal energy-saving solutions, (b) the execution and implementation and (c) the comparison of designed and actual performance as a post-implementation control. In the framework, five phases are defined in total, with three for design and two for execution and control, respectively. In the three design phases, options are generated, evaluated, and selected step by step, with appropriate multiobjective evaluation criteria covering financial as well as non-financial aspects, i.e., energy savings, GMP, worker's safety and comfort. This development extends across several phases and allows for screening for the best options using a funnel-concept; increased knowledge and level of detail is taken into account by more extensive evaluation criteria and only the most appropriate options are finally implemented. The concept of Pareto optimization as a trade-off method is used to support systematic and non-subjective decision-making. Special attention is paid to GMP regulations, which serve as a strong constraint to prevent any negative impact on product quality. In each phase the business process is supported by a so-called RACI scheme which defines roles and rights of the various stakeholders involved as responsible, accountable, consulted, and informed and which is specially adapted to the needs in the pharmaceutical industry. The proposed framework is demonstrated on the parenterals production plant at Hoffmann-La Roche in Kaiseraugst, Switzerland. In this case study, 12 energy efficiency ideas were generated and then subjected to step-by-step screening in the three design phases to successfully implement the three most promising options based on multiobjective Pareto optimization.

\section{Framework}

Figure 1 shows the proposed framework for the reduction of energy consumption in a pharmaceutical production plant. Five phases are defined for generating options comprehensively, selecting, and implementing promising ones with holistic consideration of various consequences and finally 
Fig. 1 Proposed framework for energy optimization including different stages of multiobjective evaluations and decision-making. Eng. Engineering; Ops. Operations and equipment owner; $Q A$ Quality Assurance

\begin{tabular}{|c|c|c|c|c|c|c|}
\hline \multicolumn{2}{|c|}{ Phases } & $\begin{array}{l}\text { Phase I: } \\
\text { Generate and } \\
\text { characterize } \\
\text { options }\end{array}$ & $\begin{array}{l}\text { Phase II: } \\
\text { Evaluate and } \\
\text { eliminate options }\end{array}$ & $\begin{array}{l}\text { Phase III: } \\
\text { Identify optimum } \\
\text { options }\end{array}$ & $\begin{array}{l}\text { Phase IV: } \\
\text { Implement } \\
\text { options }\end{array}$ & $\begin{array}{l}\text { Phase V: } \\
\text { Perform post- } \\
\text { implementation } \\
\text { control }\end{array}$ \\
\hline \multicolumn{2}{|c|}{ Criteria } & $\begin{array}{l}\text { - Effort } \\
\text { - Effect }\end{array}$ & $\begin{array}{l}\text { - Investment } \\
\text { - Energy } \\
\text { savings } \\
\text { - Cost savings } \\
\text { - GMP } \\
\text { - Safety } \\
\text { - Workplace } \\
\text { conditions }\end{array}$ & $\begin{array}{l}\text { - NPV } \\
\text { - GMP and } \\
\text { safety risk }\end{array}$ & $\begin{array}{l}\text { - Budget } \\
\text { - Timeline }\end{array}$ & $\begin{array}{l}\text { - Matching } \\
\text { expectations } \\
\text { - Replication } \\
\text { potential }\end{array}$ \\
\hline \multicolumn{2}{|c|}{$\begin{array}{l}\text { Investigation } \\
\text { time per option }\end{array}$} & 2-8 man-hours & 8-24 man-hours & 40-80 man-hours & Days to months & 2-24 man-hours \\
\hline \multicolumn{2}{|c|}{ Tolerance } & $\mathrm{n} / \mathrm{a}$ & $\pm 50 \%$ & $\pm 30 \%$ & $\pm 10 \%$ & $\mathrm{n} / \mathrm{a}$ \\
\hline \multirow{4}{*}{ RACl } & Eng. & $A / R$ & $\mathrm{R}$ & $\mathrm{R}$ & $\mathrm{R}$ & $A / R$ \\
\hline & Ops. & $\mathrm{R}$ & A & A & A & $\mathrm{R}$ \\
\hline & QA & C & $C$ & C & $\mathrm{C}$ & C \\
\hline & Finance & - & C & C & I & C \\
\hline
\end{tabular}

evaluating actual performance. For each phase, the following elements are defined: evaluation criteria of options, expected investigation time per option, tolerance of evaluation results and roles and rights of various stakeholders.

Each phase in Fig. 1 has a characteristic function with its own mechanisms, actions and evaluation criteria. In Phase I, possible options are brainstormed, characterized in a qualitative manner, and then passed on to Phase II without any eliminations. In Phase II, decisions are taken for the first time in order to eliminate inferior options based on quantitative evaluations, which are simple yet cover a wide range of aspects. In Phase III, the final selection of superior options is made based on detailed and focused assessment; these options are then implemented in Phase IV. Phase V is defined for comparing expected and actual performance as a postimplementation check. Information on measures not implemented is collected and stored during this phase so that the knowledge will be available for future implementation.

There are a number of key concepts realized in our framework. One is the stepwise selection approach in Phases II and III, where all options are first screened using simple indicators, and then superior ones are selected based on detailed evaluation. This "funnel" system helps companies focus only on promising options and thus deploy company resources in an effective manner. Another concept is the knowledge database in Phase V, which also contains deferred or eliminated ideas that can be used for other design cases or at a later time. This element of knowledge management provides the company with an opportunity for continuous improvement within or even beyond the site in the production network.

In parenterals production, saving energy is a crossfunctional topic and thus stakeholders from multiple business units are involved. In the field of business process management, there is a method termed RACI where the following four roles and rights are distinguished for the various players involved in an activity [20]:

- Responsible (R): The individual who actually processes the activity as a "doer"

- Accountable (A): The individual who makes the decision and provides the required resources, e.g., manpower or budget

- Consulted (C): The individual who is to be consulted before an activity proceeds to the next phase

- Informed (I): The individual who is informed about the activity but not required in the process

In this framework, RACI is applied for Engineering, Quality Assurance (QA), Operations and Finance, which are the major stakeholders throughout the five phases in Fig. 1.

\section{Phase I: Generate and Characterize Options}

The key mission of the first phase is a structured, self-driven and focused generation of options. For assistance in this challenging task, we have defined a Sankey diagram as a primary mechanism, among other conventional approaches such as Pinch technology, e.g., [21], and a literature study, e.g., $[2,16-18]$. A Sankey diagram visualizes the energy flows of a process using different thicknesses to indicate the amount of energy and different colors to represent different energy types, e.g., steam or electricity. In such a representation, one can easily identify parts of processes which are energy intensive and focus on option generation.

A hierarchical Sankey diagram is applied to parenterals processes. The following adaptations are proposed in order to support the structured generation of energy-saving measures. First, a hierarchical perspective is proposed, i.e., site, building, module, sub-module, and further down to sub-sub-module. 
Second, it is important to distinguish between modules which are active only during production (processes), e.g., filling or lyophilization systems, and ones which are always running (infrastructure), e.g., HVAC systems or water for injection (WFI) and purified water loops. This distinction makes it possible to visualize the proportion of variable and constant energy consumption for production and infrastructure, respectively, and can help determine the direction energy optimization is to take.

The generated options are then characterized with regard to the degree of effort required and the effect gained by implementation. A simple classification of "high" or "low" is used for the "effort" and "effect" categories. For this initial and qualitative assessment, various aspects need to be considered such as monetary or energy aspects, impacts on productivity, GMP relevance or workers' safety. In this phase, no decisions or eliminations are forced as too little information is available and the evaluation is based on qualitative criteria.

Experts from Engineering generate and characterize alternatives; Operations and QA can contribute to assessing the feasibility of the ideas as well as all possible consequences. Operations and Engineering are responsible and accountable for this phase, and QA needs to be consulted before Phase II is commenced. The overall duration for generating and characterizing one option is expected to be 2-8 man-hours for all stakeholders in the process (Engineering, Operations, QA, Finance and other staff). The whole landscape of generated options can be summarized in a simple two-by-two matrix showing effort and effect. This matrix is passed on to the next phase without any eliminations.
Phase II: Evaluate and Eliminate Options

The focus of Phase II is the first-time evaluation of all options generated in Phase I and elimination of inferior ones. Simple models are developed for each option which are then evaluated using the six criteria shown in Fig. 1, i.e., investment cost, energy savings, cost savings, GMP risk, safety and workplace conditions. A decision is forced for the first time so that only superior ideas are selected, as is indicated by the "D" symbol in Fig. 1.

The first task is to estimate the potential impacts of the options with modeling and simulation approaches. A few simplifications can be made in this phase, e.g., static instead of dynamic modeling, best case and worst case estimations, or the application of rules of thumb, e.g., Biegler et al. [21]. Data for these calculations can be obtained from various sources, e.g., the machine supplier, measurements [22] and retrofit data $[12,14,15]$, literature [18, 23-25], and information from other plants. Access to such data is crucial for creating shortcut models in a timely manner, which are to be refined with more elaborate data in the next phase.

The second task of Phase II is to evaluate the performance of the options using the following multiobjective indicators, which are also shown in Fig. 1 and Table 1:

- Investment in monetary units, taking into account engineering and material costs

- Energy savings in GJ equivalents per year

- Cost savings in monetary units per year with gross yield/gross benefit and increased costs such as maintenance and labor

Table 1 Evaluation indicators for GMP, safety and working conditions used in Phase II: option evaluation/elimination (with color codes). The scoring system is case-specific from the case study in this article and can vary case by case

\begin{tabular}{|c|c|c|c|c|c|c|}
\hline \multirow{3}{*}{$\begin{array}{l}\text { Quantitative } \\
\text { indicators }\end{array}$} & $1 /$ Investment & $0 \%$ & $1 \%$ & $2 \%$ & $3 \%$ & $4-100 \%$ \\
\hline & $\begin{array}{l}\text { Energy } \\
\text { savings }\end{array}$ & $0-5 \%$ & $6-10 \%$ & $11-20 \%$ & $21-50 \%$ & $51-100 \%$ \\
\hline & Cost savings & $0-5 \%$ & $6-10 \%$ & $11-20 \%$ & $21-50 \%$ & $51-100 \%$ \\
\hline \multirow{4}{*}{$\begin{array}{l}\text { Qualitative } \\
\text { indicators }\end{array}$} & Score & 1 & 2 & 3 & 4 & 5 \\
\hline & GMP & High risk & Low risk & No risk & Improvement & $\begin{array}{c}\text { High } \\
\text { improvement }\end{array}$ \\
\hline & Safety & $\begin{array}{c}\text { Very } \\
\text { negative }\end{array}$ & Negative & $\begin{array}{c}\text { No } \\
\text { change }\end{array}$ & Positive & $\begin{array}{c}\text { Very } \\
\text { positive }\end{array}$ \\
\hline & $\begin{array}{l}\text { Workplace } \\
\text { conditions }\end{array}$ & $\begin{array}{c}\text { Very } \\
\text { negative }\end{array}$ & Negative & $\begin{array}{c}\text { No } \\
\text { change }\end{array}$ & Positive & $\begin{array}{c}\text { Very } \\
\text { positive }\end{array}$ \\
\hline
\end{tabular}


In addition to these quantitative assessments, the following aspects are evaluated on a qualitative basis:

- GMP risk

- Workers' safety

- Workplace conditions

For each of these categories, an overall assessment is performed as to whether the option has a negative or positive impact during and after implementation. Such a qualitative valuation approach may vary from company to company. One approach is to use a simple scale from one to five, representing very negative, negative, neutral, positive and very positive, respectively. This scale will be demonstrated in the case study.

One technical measure can save energy in its various forms, such as electricity, steam, or cooling water; and for aggregating these different savings there are two different approaches, which are interchangeable [26]. One is to calculate the equivalent amount of reduced emissions such as $\mathrm{CO}_{2}$, e.g., Global Warming Potential (GWP) [27], while the other is to trace back to the primary energy source such as crude oil, e.g., Cumulative Energy Demand (CED) [28] and Energy Weighting Factors (EWF). The latter is a Roche internal methodology but similar concepts can be found in the EPA's national energy performance ratings [29] and in JiménezGonzález and Overcash [25], in which an LCA of the energy sub-modules is performed. The choice of aggregation method depends on the strategy of the company or the site considered. In case of Roche Engineering Basel/Kaiseraugst, the EWF is becoming the standard approach with its own site-specific values, and for each form of energy there is a converting factor for the equivalent GJ of primary energy which is easier to understand for non-experts than the $\mathrm{CO}_{2}$ equivalents.

The final task of Phase II is to select the most promising options based on six indicator results with so-called Pareto optimization, used by Hoffmann et al. [6, 7]. To make all six categories comparable, the indicator values are normalized by the maximum, i.e., best value. The normalized values are given by

$\widetilde{A}_{i}^{j}=\frac{A_{i}^{j}}{\max _{k}\left(A_{k}^{j}\right)}$

where $A_{i}^{j}$ is the value of the evaluation indicator $i$ and the idea $j$ and $\widetilde{A}_{i}^{j}$ the normalized value. For the investment cost, the inverse value $\left(A_{i}^{j-1}\right)$ can be used in order to have the best idea of the maximum. Using these normalized results, so-called Pareto optimal options are identified, i.e. a set of options which are not dominated by any other options in at least one category. Mathematically, this can be expressed as Option 2 being Pareto inferior to Option 1 if $\widetilde{A}_{i}^{1} \geq \widetilde{A}_{i}^{2}$ for all categories $i=1, \ldots n$ and $\widetilde{A}_{i}^{1}>\widetilde{A}_{i}^{2}$ for at least one $i$. Pareto optimum options are to be investigated further in the next phase. Inferior ones are eliminated here, but may be retained as ideas for reconsideration at a later point in time when sufficient resources become available. As the scoring system is based on the maximum value of an indicator the values in Table 1 can vary case by case and can be adapted as appropriate for the respective cases. To enable the timely screening of all possible ideas, the investigation time allocated is set at 8 to 24 manhours in total per idea, and the evaluation tolerance is $\pm 50 \%$. The actual work in this phase is performed by Engineering, which also includes experts in safety issues, and Operations has the right to make decision on alternatives. QA and Finance need to be consulted in assessing particularly the GMP risk and monetary performance, respectively.

\section{Phase III: Identify Optimum Options}

The goal of Phase III is to identify optimum options for the implementation, using more detailed data, models and evaluation indicators. The elaboration of the models includes replacement of static models with dynamic ones, e.g., consideration of seasonal changes and the use of measurement data instead of heuristic values.

The evaluation indicators of this phase are the net present value (NPV) as well as the combined GMP and safety risk. The NPV [21] aggregates the investment cost and cost savings criteria from Phase II with consideration of the discount rate in the future, which can be calculated by

$\mathrm{NPV}=-\mathrm{Inv}+\sum_{t=1}^{n} \frac{\mathrm{CF}_{t}}{(1+r)^{t}}$

where Inv is the investment cost, $\mathrm{CF}_{t}$ the cash flow in the year $t, n$ the number of years for the evaluation and $r$ the discount rate. Taking into account the time value of money, the NPV is one of the most detailed economic indicators of an investment project and thus widely used in industry. Values of $t, n$ and $r$ are typically defined in companies as a standard, while Inv and CF in a year $t$ are calculated based on the model. All evaluation categories considered in Phase II are reflected in the NPV: Inv also covers costs for preventing any issues associated with safety, GMP or working conditions; CF reflects benefits achieved by monetary and energy savings.

An analytical method termed Failure Mode and Effect Analysis (FMEA) is applied for quantifying risks related to different kinds of aspects, especially safety and GMP. It is one of the recommended risk analysis methods from the health authorities in the ICH Q9 [30] and broadly used in the pharmaceutical industry. In an FMEA, various failure modes of a process such as "valve defect" are identified and then the risk is assessed for each failure mode, resulting in the risk priority number (RPN) which is calculated by 
$\mathrm{RPN}=$ Severity $\times$ Occurrence $\times$ Detectability

where Severity accounts for a consequence of the failure mode, such as potential impact on product quality; Occurrence for the frequency of the failure, such as once a year; Detectability for the ability to detect the failure, such as annual maintenance or revalidation. Usually, an integer classification such as 2, 4, 6, 8, 10 is applied for each term with ascending importance, whereas scaling differs depending on company internal guidelines. The FMEA characterizes each option with different failure modes and corresponding RPN numbers, and a maximum RPN of an option is selected to represent the worst consequence.

Using the results of these two indicators, Pareto optimal options are identified considering the NPV and RPN as objective functions for maximization and minimization, respectively. Based on this Pareto analysis, the final decision with consideration of available resources and timing is made as to which option(s) to implement. In several situations, options that are identified as Pareto inferior can also be selected for implementation or, in the opposite case, some of the Pareto optimal ideas could be deferred. Compared to the previous phase, the accepted error tolerance of the evaluation results is reduced from \pm 50 to $\pm 30 \%$. All activities in this critical phase, i.e., modeling, evaluation and decision-making, would require 40 to 80 man-hours per option performed by various experts. The RACI structure is the same as in Phase II.

\section{Phase IV: Implement Options}

The options selected in the previous phase are finalized as a design and implemented in Phase IV. The evaluation indicators from the previous phase, i.e., the NPV and RPN, are updated with new information, and the evaluation tolerance is further reduced from \pm 30 to $\pm 10 \%$. Financing has to be planned in advance and additional budgets dedicated, for example, to quality, workers' safety and workplace conditions, considered. The appropriate timing needs to be defined which considers production and maintenance schedules. While operational changes, such as the adjustment of parameters, can be performed parallel to production, hardware changes, such as the replacement of equipment, may well require interrupting production for some time. Major changes may be necessary during plant maintenance especially if the infrastructure (e.g., media supply or air conditioning) is to be modified since the entire production building is affected. In most cases, GMP-related activities are to be performed along with implementation, such as technical change management, qualification \& validation, documentation, SOP updates and training. Performance of the implemented options needs to be monitored intensively in the initial period, and special attention should be paid to any unforeseen events or reactions. When initial troubles are too difficult to solve, it might become necessary to make a decision to change or stop implementation. Learning from failures, though, is important from the perspective of knowledge management, and thus such experience is to be passed on to the next phase: postimplementation control. Depending on the option, e.g., software versus hardware changes, this phase can take from days to months. In the RACI, R and A remain the same from Phase III, while QA is consulted for the change approval and Finance is informed of the outcome.

\section{Phase V: Perform Post-Implementation Control}

The main aim of this phase is to maintain the continuity of the improvement activity by comparing expected and realized performances and identifying further improvement opportunities. To obtain the big picture of the "before and after" situation, refreshing the Sankey diagram initially created in Phase I is a useful approach. Furthermore, some of the evaluation indicators in Phases II and III, such as energy savings or the RPN, are updated for a detailed view. If there is a gap between the expectation and the result, a root cause analysis should be performed to remove the source of the problem rather than attempt additional temporary solutions. Deferred or eliminated options in Phases II and III can be reconsidered in case greater energy savings are needed in order to reach a certain target value. Such deferred or eliminated options may be suitable for implementation in other plants and sites. Furthermore, the framework itself can be revised and improved by, for example, adding new or modifying indicators, adjusting the working hours and RACI case- or companyspecific. This kind of information exchange and knowledge management within the company is an essential part of continuous improvement not only for energy savings but also for other themes in general, in particular in the Pharmaceutical Quality System [31]. This phase takes 2-24 h, depending on the option as simple feedback is less time intensive than a measurement campaign. This activity is performed by Operations and Engineering, who are also accountable for the outcome, and QA as well as Finance are consulted to draw conclusions.

\section{Roche Parenterals Production Kaiseraugst}

Located on Hoffmann-La Roche Ltd.'s site in Kaiseraugst, Switzerland, PKau (Parenterals Production Kaiseraugst) is one of the newest facilities for manufacturing sterile drug products in Europe. The building, shown in Fig. 2a, has a gross floor area of 12,900 $\mathrm{m}^{2}$ and consists of four production modules: compounding, liquid vials (shown in Fig. 2b), lyophilized vials, and prefilled syringes. Design activities started in 2006, followed by the construction of the building and machine installation, which were completed in 2009. The total investment amounted to approximately CHF 200 million. 
Fig. 2 Introducing parenterals production Kaiseraugst of $F$. Hoffmann-La Roche Ltd.: a facility exterior; $\mathbf{b}$ filling line of liquid vials using isolator technologies are found in first floor

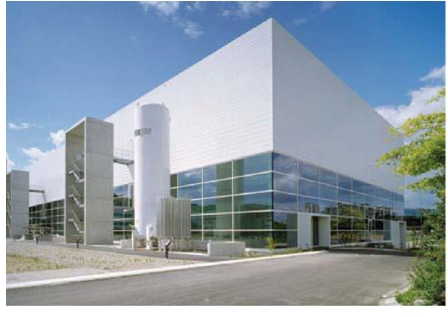

(a)

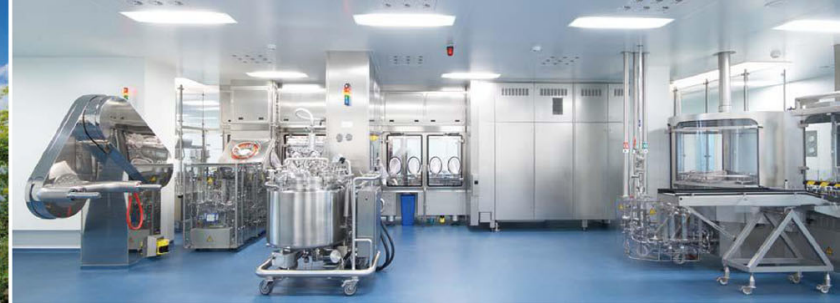

(b)
Since then, various start-up activities have been performed, such as qualification/validation, creating SOPs, training and hosting health authority inspections. Commercial production started in 2012, and during the ramp-up to 2014, PKau will play a major role in Roche's global drug product manufacturing network.

PKau features a few technical highlights. For example, isolator and Restricted Access Barrier System (RABS) technologies are applied in aseptic filling processes for the prevention of product contamination by minimizing particle concentration and excluding microorganisms. A grade D environment is maintained for the isolators/RABS, laboratories, offices and storage rooms, which is where most of the supporting activities take place. For the most part, the energy and media systems are designed redundantly so that production can proceed even if the energy and media systems are interrupted. Each module has its own independent HVAC system, which means that modules can be shut down individually for maintenance while others remain in operation. At the moment all the products are biologics, i.e., the active pharmaceutical ingredients are based on biologically produced proteins, which require strict control of the production environment.

Parallel to the implementation of these technologies to maximize GMP compliance and meet diverse customer requirements, e.g., Japan Quality [32, 33], deliberations were also made with regard to energy savings. External experts were also involved, and various ideas were generated and implemented, such as high-efficiency light bulbs, heat recovery systems using condensed steam, and top-performance ventilation engines. During the start-up, however, several opportunities were discovered for further minimization of energy consumption. PKau management has a vast interest in continuous improvement [34] and has therefore decided to improve energy efficiency within the building along with the method described in the "Framework" section.

\section{Case Study}

Phase I

To guide the option generation process, the hierarchical Sankey diagram shown in Fig. 3 was created according to the description in "Phase I: Generate and Characterize Options". The preliminary version of Fig. 3 was presented elsewhere [35]. It visualizes the five energy sources provided from the Kaiseraugst site to the PKau building (Fig. 2a) in different colors as well as their expected consumptions
Fig. 3 Sankey diagram of PKau process systems with hierarchical perspective. At the module level, units included in the production processes are performed batchwise, while the ones in the infrastructure are running continuously

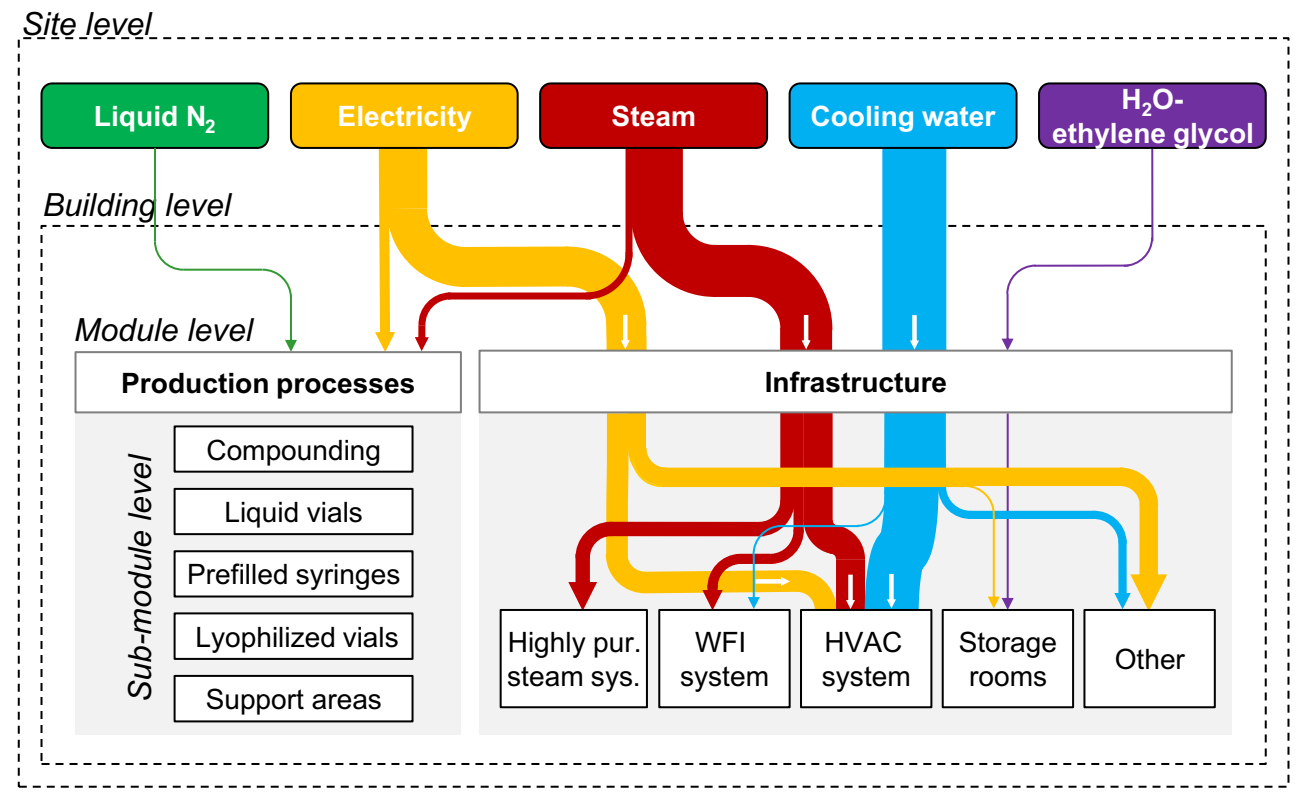


relative to each other for 2012: liquid nitrogen, electricity, steam, $\mathrm{H}_{2} \mathrm{O}$-ethylene glycol $\left(-8{ }^{\circ} \mathrm{C} /-3{ }^{\circ} \mathrm{C}\right.$ cooling loop $)$ and cooling water $\left(6^{\circ} \mathrm{C} / 12{ }^{\circ} \mathrm{C}\right.$ cooling loop).

It is possible to identify various proportions of energy consumption from Fig. 3: electricity, steam, and cooling water account for most of PKau's energy consumption; liquid nitrogen and $\mathrm{H}_{2} \mathrm{O}$-ethylene glycol, on the other hand, make up only a fraction of this. This fact led to energy efficiency options which focus on electricity, steam, and cooling water. At "module level", it is evident that most of the energy is consumed in infrastructure and only a fraction in processes. A sensitivity analysis has proven that this will also be the case in the future following the start-up phase. This situation led to the decision to focus on generating energy efficiency options within the scope of the infrastructure rather than within the scope of the production process. At the sub-module level of the infrastructure only the largest consumers are shown, i.e., highly purified steam, WFI, and HVAC systems. The latter is the most energy-intensive sub-module because in parenterals production high air change rates $\left(20 \mathrm{~h}^{-1}\right.$ for a grade D environment in the rooms around Production; $10 \mathrm{~h}^{-1}$ in other grade $\mathrm{D}$ rooms) with an exactly specified room temperature and humidity are required to minimize particles and microbial concentration. This has also been described for other pharmaceutical manufacturing buildings [2, 17]. The WFI and highly purified steam systems are $100 \%$ redundant and thus have a higher base consumption.

According to the RACI scheme in this phase, an expert group from Engineering, Operations, and QA generated and characterized the 12 feasible options in Phase I. These options are shown in Fig. 4 and described below with their Phase I evaluations.

- Option 1 is the installation of the enthalpy control system shown in Fig. 5. It is an energy monitoring and control system for the HVAC system to minimize energy costs, i.e., measuring the enthalpy of the outside and the exhaust air to find the optimum mixture of recirculated air system, heat recovery system, heating, cooling and humidification. The effort required to install such a system would result in the highest cost savings of all measures. More detailed information for this option can be found in the Appendix.

- In Option 2, the WFI subloop was changed from permanent cooling to on-demand cooling by training the operators to stop the system more effectively. In the WFI subloop $80^{\circ} \mathrm{C}$ WFI is cooled to $20^{\circ} \mathrm{C}$ for compounding and heated back up to $80^{\circ} \mathrm{C}$ for re-introduction into the WFI main loop. Reducing the operating times of this system saves steam and cooling water. The effort, characterized as low, involves training the operators to use the stop button more efficiently. The system is redundant so training is only required once. The effect: unnecessary cooling can be reduced, which results in advantages in terms of energy and economy.

- In Option 3, the pipes between the WFI distillation columns in PKau, the column heads and the WFI storage tank covers are insulated. As an effect, this improvement will
Fig. 4 Characterizing generated

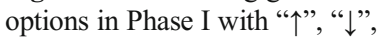
$\mathrm{CW}$ and elec. representing increase in consumption, reduction in consumption, cooling water and electricity, respectively

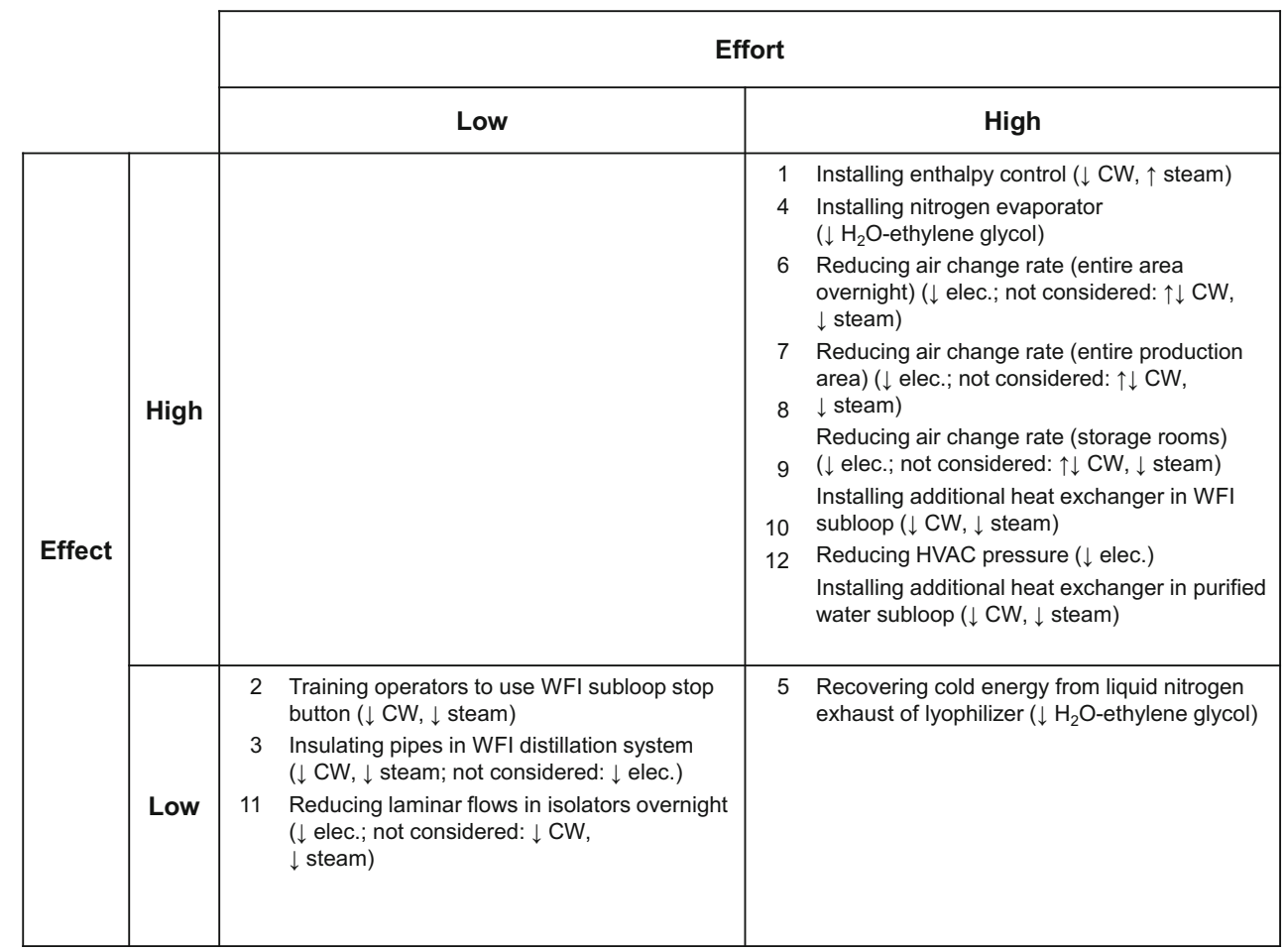


Fig. 5 Option 1: enthalpy control system as a representative generated option

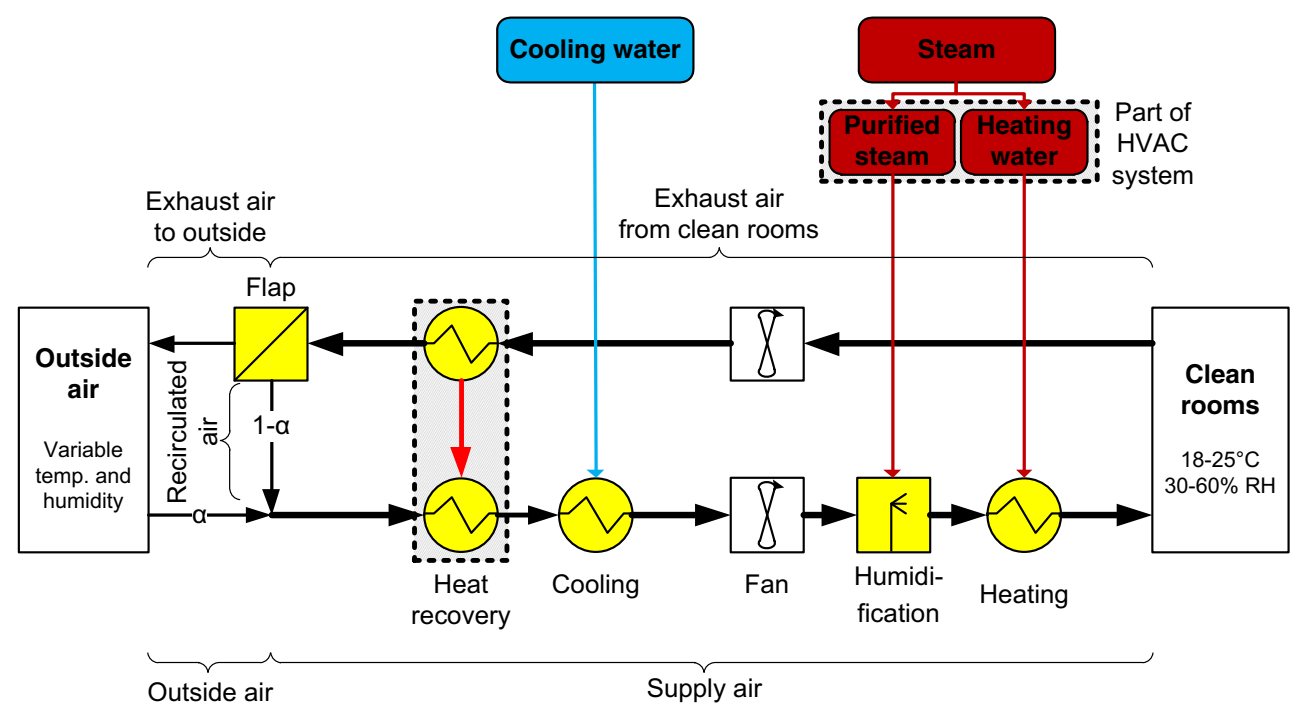

reduce the loss of heating energy of the columns and cooling energy for maintaining the room temperature of the room where the columns are located. Additional effects include improved safety as a result of fewer hot surfaces and improved workplace conditions as a result of the lower room temperature.

- In Option 4, energy from the nitrogen evaporator for the gaseous nitrogen supply is used. In the current state the cold energy is blown into the environment and not collected. Option 5 uses the cold energy after the phase change that is not consumed by the lyophilizer. In both options, the cooling energy would be collected in heat exchangers and returned to the $\mathrm{H}_{2} \mathrm{O}$-ethylene glycol loop, reducing the cooling requirements of the building.

- In Options 6-8, the air change rate is reduced in different scenarios to save electricity, purified steam and cooling water. Option 6 reduces the air change rate in the whole building overnight by introducing a system with two operation modes, i.e., a normal mode and a standby mode for night-time use. The air change rate would be permanently reduced with Option 7 in the production area and with Option 8 only in the storage rooms. All three options were calculated for a reduction of the air change rate of $10 \%$, resulting in an attractive reduction (approx. $30 \%$ ). As it can be seen in the Sankey diagram in Fig. 3, a huge part of PKau's electricity is consumed by the HVAC system, which gives this option a high potential for savings. Consequently, it is listed as a high-level effect in Fig. 4. The changed cooling and humidification performance was not considered in Phases I+II as this would require exact simulations, which were not carried out until Phase III.

- In Option 9, a new heat exchanger is installed between the valve and the heater in the WFI subloop, saving steam, and cooling water. The idea of this measure is transferred to Option 12 where purified water is heated from $20^{\circ} \mathrm{C}$ to $80^{\circ} \mathrm{C}$.

- Option 10 reduces the HVAC pressure by $1 \%$ from 600 to $594 \mathrm{~Pa}$ overpressure to save electricity since the pressure is set higher than the simulated values to prevent problems with pressure drops.

- Option 11 reduces laminar flows in the isolators for aseptic filling by $10 \%$ from 0.450 to $0.405 \mathrm{~m} / \mathrm{s}$ overnight when no production takes place, including the assumptions for Options 6-8.

Figure 4 shows the summary and the classification of the options in Phase I in a matrix showing the effort required and effect of the implementation. No option was classified in the category of low effort with high effect, which would have been the most preferable case, suggesting that the most promising options have already been implemented and that Engineering worked well during the design stage. Eight options were evaluated as high effect with high effort, e.g., Option 1's level of investment is high and GMP problems are expected. The effect: a high level of financial and energy benefit because the HVAC system is the largest energy consumer in the building (see Fig. 3). The ideas on adjusting the air change rate, i.e., Options 6 to 8 , would require clarification on GMP risk, which was ranked as high effort, while the effect could be high energy and cost savings. Similarly, Options 10 and 11 are GMP relevant as the ventilation systems are changed. Options 4 and 5 are technically feasible and provide high financial and energy savings. However, the energy could only be recovered inconstantly since the energy can only be used when the system is running (not periodic, on/off operation). Only Option 5 was classified in the low effort and low effect category as it could be implemented without high GMP risk and investment, but resulted in low savings. 
Although no decision or selection is forced in Phase I, a qualitative classification of the priorities was made based on Fig. 4. The preferred optimum is the low effort/high effect category, followed by both the low effort/low effect and high effort/high effect options, whereas the option in high effort and low effect is third priority. Thus, there is no option ranked as first priority, the second priority ones were tackled first in Phase II and then the third priority one. In our case study, no measure was eliminated in this phase because no measure was considered to be completely infeasible.

\section{Phase II}

After each option was designed as described in "Phase II: Evaluate and Eliminate Options", each one was evaluated for the first time according to the multiobjective indicators of Phase II in Fig. 1, and then normalized using Eq. 1. For the qualitative evaluation the scoring system for the GMP, workers' safety, and workplace conditions in Table 1 is used. For the selection of the best and elimination of inferior options, two different but equivalent visualization aids were used: a spider diagram (Fig. 6) and "heat map" (Table 2). In both, values of $A$ are reported in the range of $[0,1]$ and the investment criterion is inverted to have the maximum and preferred values and thus best option as the highest value.

The spider diagram in Fig. 6 is proposed for the selection of the best options in Phase III and was preferably used in this case study. The evaluation indicators are shown from 0 to $100 \%$ on the axis.

The heat map in Table 2 is a further tool for the identification of the Pareto optima using the color scheme of Table 1 to highlight the differences between the normalized values with regard to the various options and criteria. For this project, the absolute values were assigned a color scheme and then this color scheme was also used for the relative values. The color scheme in Table 1 can be individually adapted to suit the company's requirements.

The best and worst option in each category can be identified from Fig. 6 and Table 2. This information was crucial for the following Pareto optimization:

- In the $1 /$ Investment criterion, Option 2 is the best because of the short training time for the operators while Option 6, which requires drastic changes in the ventilation system, is rated as the worst.

- The highest energy savings are achieved by Option 6 since the ventilation system is the largest consumer in the building. On the other hand, Options 5 and 12 have the lowest savings since there is only little potential from the nitrogen exhaust air and the purified water subloop is rarely used.

- The highest cost savings result from Option 1, the enthalpy control system, which saves immense amounts of cooling, and the lowest from Option 12 as the purified water subloop is rarely used.

- In the GMP criterion, seven ideas are considered to be "high risk" or GMP $=1$, which is $33 \%$ in Fig. 6 and Table 2 because the maximum score was 3, i.e., none of the options improved GMP conditions. Options 6-8 to reduce the air change rate either violate GMP regulations or their effects on particle and microbiological concentrations in clean rooms cannot be estimated, which is also valid for Option 11 and the isolators, respectively. For Options 9 and 12 it remains unknown if the GEP (good engineering practice) of having turbulent flow in the whole system will still be valid after implementation and for Option 10 it cannot be guaranteed that the pressure differences between the zones can be maintained.

- Safety is increased by Option 3 because hot pipes, tank covers and other hot surfaces are insulated, significantly lowering the probability of burns.

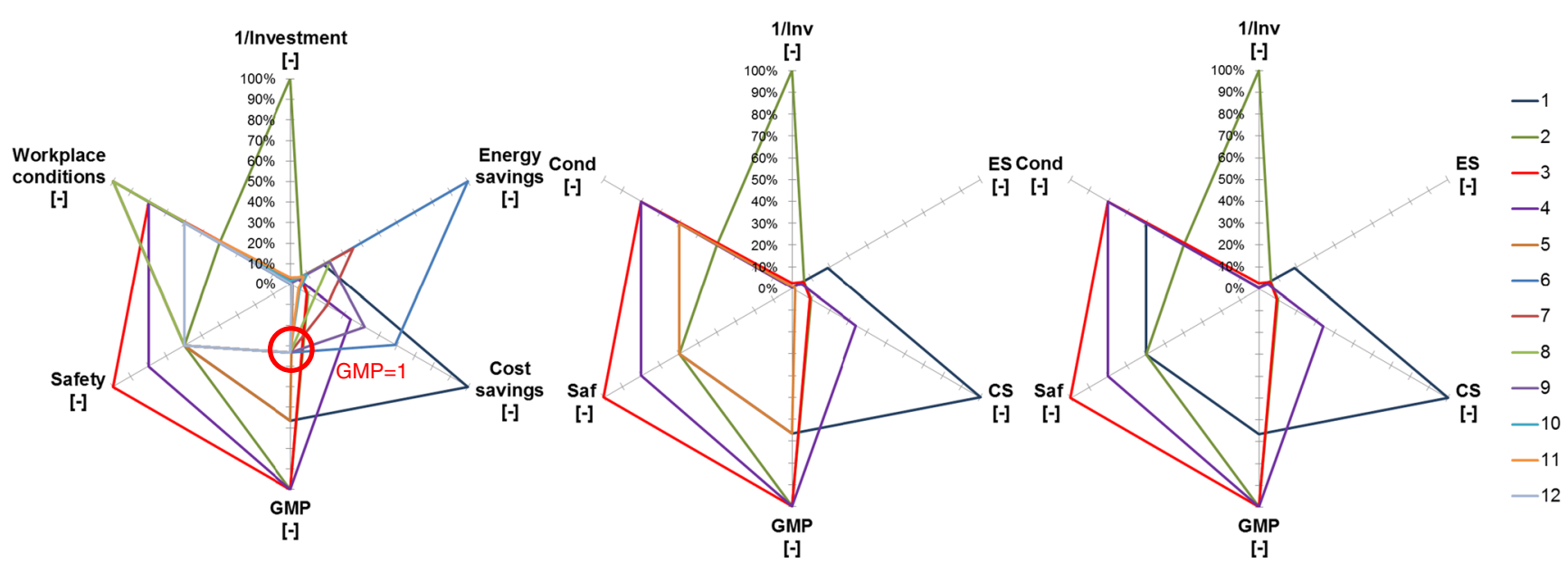

Fig. 6 Evaluating and eliminating options in Phase II: a normalized indicator values $\widetilde{A}$ and for investment $\widetilde{A}^{-1}$ of all 12 options; b result after eliminating options with GMP $=1$ (high risk on product quality); $\mathbf{c}$ Pareto optimal options 
Table 2 "Heat map" representation of evaluation results with values of $\widetilde{A}$ and $\widetilde{A}^{-1}$ based on color code for evaluation indicators used in Phase II

\begin{tabular}{|c|c|c|c|c|c|c|c|}
\hline Index & Measure & $\begin{array}{c}1 / \operatorname{lnv} \\
{[-]}\end{array}$ & $\begin{array}{c}\text { Energy } \\
\text { savings } \\
{[-]}\end{array}$ & $\begin{array}{c}\text { Cost } \\
\text { savings } \\
{[-]}\end{array}$ & $\begin{array}{c}\text { GMP } \\
{[-]}\end{array}$ & $\begin{array}{c}\text { Safety } \\
{[-]}\end{array}$ & $\begin{array}{c}\text { Workplace } \\
\text { conditions } \\
{[-]}\end{array}$ \\
\hline 1 & Installing enthalpy control system & $1 \%$ & $19 \%$ & $100 \%$ & $67 \%$ & $60 \%$ & $60 \%$ \\
\hline 2 & Training operators to use WFI subloop stop button & $100 \%$ & $6 \%$ & $10 \%$ & $100 \%$ & $60 \%$ & $40 \%$ \\
\hline 3 & Insulating pipes in WFI distillation system & $3 \%$ & $6 \%$ & $9 \%$ & $100 \%$ & $100 \%$ & $80 \%$ \\
\hline 4 & Installing nitrogen evaporator & $1 \%$ & $5 \%$ & $34 \%$ & $100 \%$ & $80 \%$ & $80 \%$ \\
\hline 5 & Recovering cold energy from liquid nitrogen exhaust of lyophilizer & $1 \%$ & $1 \%$ & $2 \%$ & $67 \%$ & $60 \%$ & $60 \%$ \\
\hline 6 & Reducing air change rate (entire area overnight) & $0.1 \%$ & $100 \%$ & $59 \%$ & $33 \%$ & $60 \%$ & $100 \%$ \\
\hline 7 & Reducing air change rate (entire production area) & $1 \%$ & $35 \%$ & $21 \%$ & $33 \%$ & $60 \%$ & $100 \%$ \\
\hline 8 & Reducing air change rate (storage rooms) & $1 \%$ & $23 \%$ & $14 \%$ & $33 \%$ & $60 \%$ & $100 \%$ \\
\hline 9 & Installing additional heat exchanger in WFI subloop & $1 \%$ & $22 \%$ & $42 \%$ & $33 \%$ & $60 \%$ & $60 \%$ \\
\hline 10 & Reducing HVAC pressure & $2 \%$ & $9 \%$ & $5 \%$ & $33 \%$ & $60 \%$ & $60 \%$ \\
\hline 11 & Reducing laminar flows in isolators overnight & $3 \%$ & $8 \%$ & $4 \%$ & $33 \%$ & $60 \%$ & $60 \%$ \\
\hline 12 & Installing additional heat exchanger in purified water subloop & $1 \%$ & $1 \%$ & $1 \%$ & $33 \%$ & $60 \%$ & $60 \%$ \\
\hline
\end{tabular}

- The workplace conditions in Options 6-8 are positively affected as the reduction of the air change rate also reduces the noise and the draftiness of the ventilation system.

For a systematic and automated selection process of the most promising and the elimination of non-suitable options, the following elimination algorithm was used in this case study:

1. Only measures evaluated in each criterion are considered.

2. Options that are "high risk" for GMP conditions $(\mathrm{GMP}=1)$ are eliminated.

3. Pareto analysis of the remaining options: Pareto optima are passed on to Phase III as first priority; Pareto inferior options remain in this phase and are passed on at a later point in time as second priority and stored in the knowledge database.

For execution of this algorithm in this case study, only the 12 fully evaluated measures were considered in the spider diagram in Fig. 6 and the heat map in Table 2 as well as for Pareto optimization. Of the 12 fully evaluated options in Fig. 6a, the 7 options with GMP $=1$ (red circle) were eliminated because in the pharmaceutical industry, no "high risks" for GMP conditions are desired. Of the remaining Options $1-5$ in Fig. 6b, Option 5 can be identified as Pareto inferior as this option is equal to or worse than Options 3 and 4. Figure 6c shows Pareto optima 1-4. These optima are all equal, accepted, and passed on to Phase III for a more delineated design and evaluation. The same elimination process can be performed using the heat map shown in Table 2, where the measures have first been sorted by Pareto optima, Pareto inferior and GMP $=1$ options for better readability. The latter options can be identified as they are highlighted in red and are therefore eliminated. Looking at Options $3-5$, one can see that Option 5 is always equal to or worse than Options 3 and 4, making it Pareto inferior and thus deferred. Option 5 was already evaluated as Pareto inferior in Phase I, and this rough classification is later verified.
Phase III

The four remaining options from Phase II were delineated more extensively and then evaluated according to the evaluation criteria in Phase III: the NPV and risk regarding safety and GMP.

In Fig. 7, the results of the above evaluation of all 4 options are shown with the NPV on the abscissa and the Maximum RPN from the FMEA on the ordinate. The NPV is calculated using Eq. 2, with the number of years $n$ and the discount rate $r$ taken from a company internal guideline. It can be seen in Fig. 7 that no option has a negative NPV. Option 1 has the highest one as it saves large amounts of energy at a comparably low investment cost, whereas Option 3 has the lowest NPV because of its lower benefit. The GMP and safety risk are combined and evaluated by means of an FMEA according

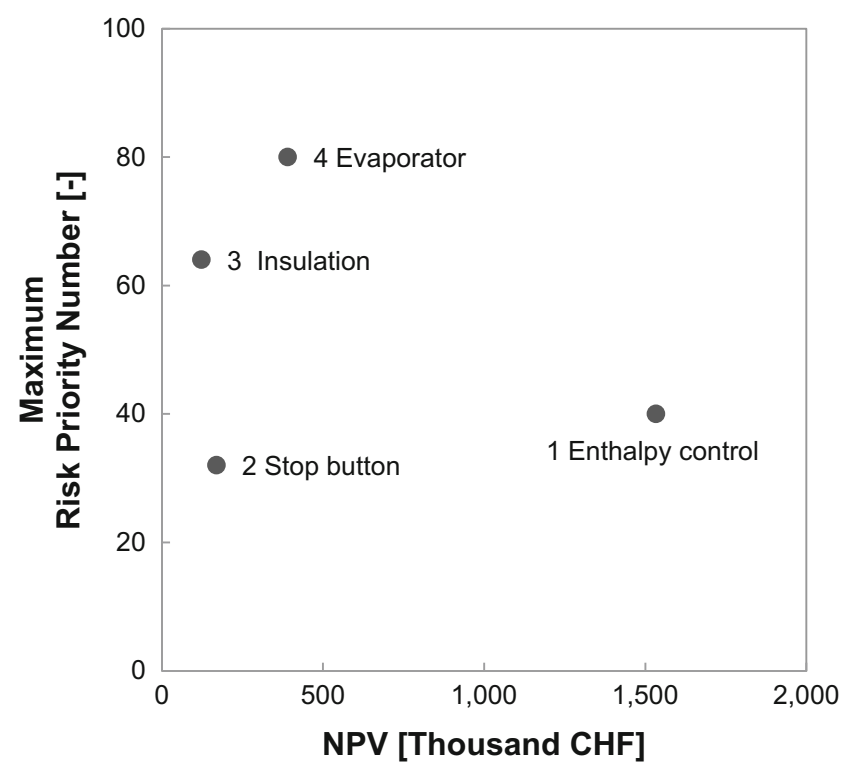

Fig. 7 Evaluation results of the options in Phase III for the identification of Pareto optima 
to "Phase III: Identify Optimum Options" and a company internal guideline for scaling Severity, Occurrence, and Detectability. For example, the maximum risk of Option 1 is represented by a failure mode where the temperature of the clean room is influenced by outside temperature fluctuations. According to the Roche guideline, a Severity of 2 was achieved because the temperature in the HVAC system does not have an influence on product quality since the isolator's after-cooling protects the product. As temperature fluctuations occur constantly, no prevention measures were chosen, resulting in an Occurrence of 10. Because the clean room monitoring system as well as the temperature control system detect any problems with regard to temperature, Detectability was set to 2. The RPNs were then calculated using Eq. 3, resulting in an RPN of 40 for the above-mentioned failure mode in Option 1. The maximum value of all RPNs is termed "Maximum RPN" and it is the one with the greatest adverse effect on product quality or workers' safety. In the case of Option 1, the Maximum RPN was the above-mentioned example with the temperature fluctuations. For the other three options, the same procedure was applied, resulting in the highest value for Option 4 because of the difficult implementation and lack of experience. Because of this uncertainty combined with high risk, this option was rejected by QA and not passed on for implementation. It is stored as an idea for future implementation.

A maximum NPV and minimum GMP and safety risk is desired, thus Options 1 and 2 are Pareto optima because they superimpose Option 3 and the eliminated Option 4 in both criteria. As sufficient engineering capacity and budget was available, all options were passed on with Options 1 and 2 as priority 1 and Option 3 as priority 2 .

\section{Phase IV}

In Phase IV after finalizing the design, the budget and timeline were set up and the three options passed on from Phase III were implemented.

The implementation itself and its results as well as postimplementation control for the four options in Phase III are different for each measure: the enthalpy control system (Option 1) was implemented in one of the HVAC systems because the timeline was suitable, and it is running according to all regulations. The stepwise introduction in the other systems will be performed after enough experience has been gained from this first system and after its effectiveness and feasibility have been confirmed. Operators have been trained for Option 2, but it has not been realized to full our satisfaction as the button could be used even more efficiently. Option 3 has been implemented with complete success as safety has increased drastically because most of the hot surfaces are covered and the temperature in the room decreased significantly, improving workplace conditions for the operators' benefit.

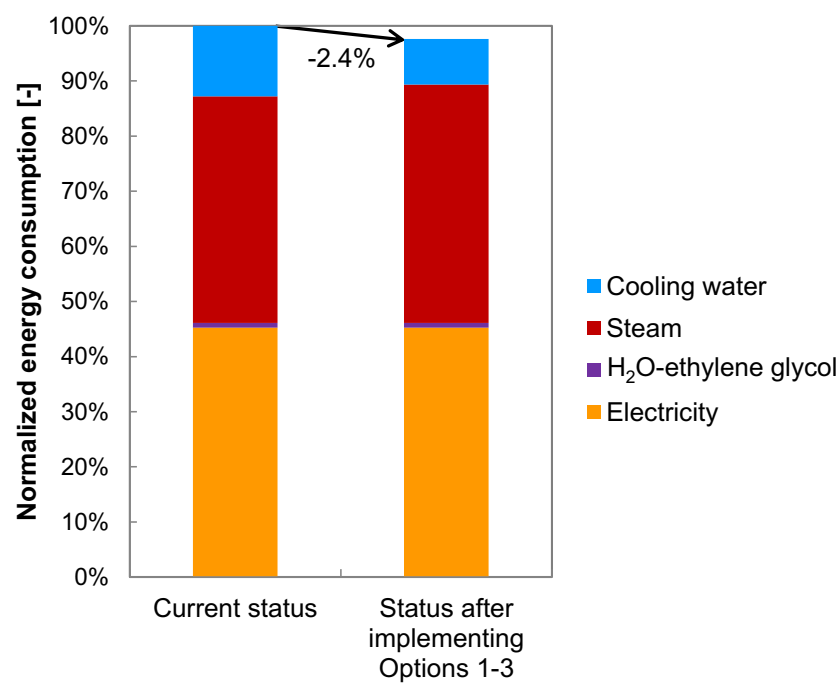

Fig. 8 Comparing energy consumption before and after implementing Options 1-3

\section{Phase V}

Phase V analyzed the results of the implementation, the extent to which they meet expectations and the replication potential. Within the scope of knowledge management, options which are not passed on/eliminated are stored together with the experiences made in connection with implementation. All of these options are revisited and reinvestigated, and may be implemented.

Figure 8 shows the business results of this case study as unweighted energy consumption using a "before and after" comparison. After the implementation of the proposed Options $1-3$, PKau energy consumption is reduced by $2.4 \%$ using the EWF and $12.9 \%$ in unweighted numbers. The preliminary results reported elsewhere [35] have been updated. Most of the reduction is with regard to cooling water, which is energy non-intensive to produce as a free-cooling unit can be used $(\mathrm{EWF}<1 \mathrm{GJ} / \mathrm{GJ})$, whereas more steam, which is energy intensive, is needed for the humidification (EWF $>1$ GJ/GJ).

In Phase $\mathrm{V}$, the replication potential of each alternative was evaluated. Option 1, the enthalpy control system, already uses experience from other buildings and the lessons learnt from this implementation will be transferred to other projects. Option 2 is PKau-specific and cannot be replicated in other buildings; however, it is possible to apply the concept if the purified water subloop is used more often. The positive experience and results of Option 3 can be easily transferred to other buildings and sites. For Option 4, the replication potential is limited since it has not been implemented for PKau, but possibilities for other buildings and sites will be investigated. The working processes and outcomes of the project were reported to the upper management of Engineering in order to ensure the possibility of implementation. 


\section{Conclusions and Outlook}

In this article, we have presented a novel framework for energy optimization in a pharmaceutical plant. Its overall purpose is to (a) support a design set of optimal energysaving solutions, (b) execute the implementation and (c) compare the plan and the result as post-implementation feedback. The possible energy efficiency measures are first generated and then the best ones are selected for implementation in a stepwise screening process. Multiobjective evaluation criteria covering financial and non-financial aspects are defined to account for the comprehensive benefits of energy conservation. To support a systematic and non-subjective decisionmaking process, Pareto optimization is used as a way of allowing a trade-off between the various multiobjective evaluation criteria. The framework is based on specific elements for the pharmaceutical sector, e.g., GMP, risk analysis and the stakeholders in pharmaceutical production. During the whole process a RACI scheme defines the rights and responsibilities of the various stakeholders. The importance of GMP in the pharmaceutical industry is reflected by the use of risk analysis tools, e.g., the industry standard FMEA, to discover and prevent any negative impact on product quality.

This framework was executed in an industrial case study on the parenterals production factory of Hoffmann-La Roche Ltd. in Kaiseraugst, Switzerland, proofing the concepts for screening measures, the stepwise selection and the exact definition of roles and responsibilities. Starting with 12 generated energy efficiency options, the 3 best ones were chosen using a stepwise selection process and implemented. These three options show the best multiobjective performance and do not have any negative impact on GMP regulations or quality. This screening concept made it possible to rapidly evaluate many measures and only select the best ones. In cases of limited engineering capacity or budget, this may be important because resources such as capacity or budget can be used very efficiently by focusing on the measures individually. To support this generation and selection process, various visualization methods were presented. The non-selected ideas are stored in a knowledge database so that the idea for the measures and the invested time are not lost. According to the concept of continuous improvement, these ideas can be reconsidered at any time for future implementation. The business results showed that a reduction of energy consumption of $2.4 \%$ using the EWF concept is even possible in a brand new factory. During the execution of the framework, the defined roles and rights for all stakeholders from the RACI scheme facilitated effective communication among the teams of QA, Operations, Engineering, and Finance.

The framework is kept generic so that it can be applied to other plants, sites and companies independent of the technology used to manufacture drug substance or drug product. This means that only minor modifications are required for adaptation to different situations, e.g., RACI in particular. It may also be possible to use the framework for media optimization and in other GMP-relevant industries, e.g., the medical technology or food industries as they have similar requirements. In the future, these applications will have to be tested in further industrial case studies.

Acknowledgments The authors are grateful for the discussions with Dr. Stavros Papadokonstantakis and Prof. Konrad Hungerbühler at ETH Zurich. Part of this work was financially supported by Grants-in-Aid for Young Scientists (Start-up) No. 25889016 from the Japan Society for the Promotion of Science.

\section{Appendix}

Option 1 is the installation of the enthalpy control system shown in Fig. 5. The heat recovery unit consists of two heat exchangers: at outside temperatures of $<12{ }^{\circ} \mathrm{C}$ the heating energy from the exhaust air from the clean rooms can be transferred to the supply air; at hot outside temperatures $>30{ }^{\circ} \mathrm{C}$ cooling energy from the exhaust air from the clean rooms can be transferred to the supply air. Because of hygienic aspects, an outside air to supply air ratio of $\alpha \geq 0.3$ has to be guaranteed. The recirculated air system can recirculate $1-\alpha$ of the exhaust air to save heating, cooling and humidification while $\alpha$ comes from the outside air and is used for the supply air which flows through the second part of the heat recovery unit, the cooling unit, the fan, the humidification unit and the heating unit. The conditions in the clean room with 30-60\% $\mathrm{RM}$ (relative humidity) and $18-25^{\circ} \mathrm{C}$ are constraints from the GMP regulations that have to be fulfilled at all times and reduce the degree of freedom in the operation. Without the enthalpy control system, the air recirculation is set to a fixed value and the heat recovery unit works at an energetically nonoptimum operating point. The enthalpy control system collects the temperature and humidity data from the outside air and the exhaust air from the clean rooms and using the heat recovery and the recirculated air system, it tries to reduce energy consumption in the cooling, humidification and heating unit by calculating the enthalpy of different operating modes and using the optimum one. The main benefit of the enthalpy control system is generated in spring and autumn when the outside air has a humidity and temperature similar to clean room conditions because then it is possible to operate the HVAC system without heating, cooling and humidification by using $100 \%$ outside air and the heat recovery unit for the air conditioning. In winter and summer, the maximum recirculating air ratio of $\alpha$ has to be used. In winter the air is dry and cold, thus a large amount of energy has to be used for heating and humidification, and in summer the air is hot and humid, thus a large amount of cooling energy for cooling and 
dehumidification is lost. For this option a trade-off between cooling water and purified steam has to be made because a lot of cooling can be saved whereas more purified steam for humidification is consumed.

\section{References}

1. Bundesamt für Energie. Überblick über den Energieverbrauch der Schweiz im Jahr 2010. Ittigen (Switzerland): BBL/ Bundespublikationen; 2010.

2. Galitsky C, Chang S, Worrell E, Masanet E. Energy efficiency improvement and cost saving opportunities for the pharmaceutical industry: An ENERGY STAR ${ }^{\circledR}$ Guide for energy and plant managers. Berkeley: Ernest Orlando Lawrence Berkeley National Laboratory; 2008.

3. Dow Jones Sustainability Indexes. http://www.sustainabilityindexes.com/. Accessed 19 Sept 2012.

4. Sugiyama H, Fischer U, Hungerbühler K, Hirao M. Decision framework for chemical process design including different stages of environmental, health and safety assessment. AIChE J. 2008;54:103753.

5. Chen H, Shonnard DR. Systematic framework for environmentally conscious chemical process design: early and detailed design stages. Ind Eng Chem Res. 2004;43:535-52.

6. Hoffmann VH, McRae GJ, Hungerbühler K. Methodology for earlystage technology assessment and decision making under uncertainty: application to the selection of chemical processes. Ind Eng Chem Res. 2004;43:4337-49.

7. Hoffmann VH, Hungerbühler K, McRae GJ. Multiobjective screening and evaluation of chemical process technologies. Ind Eng Chem Res. 2001;40:4513-24.

8. Albrecht T, Papadokonstantakis S, Sugiyama H, Hungerbühler K. Demonstrating multi-objective screening of chemical batch process alternatives during early design phases. Chem Eng Res Des. 2010;88: 529-50.

9. Heinzle E, Weirich D, Brogli F, Hoffman VH, Koller G, Verduyn MA, et al. Ecological and economic objective functions for screening in integrated development of fine chemical processes. 1. Flexible and expandable framework using indices. Ind Eng Chem Res. 1998;37: 3395-407.

10. Koller G, Weirich D, Brogli F, Heinzle E, Hoffmann VH, Verduyn MA, et al. Ecological and economic objective functions for screening in integrated development of fine chemical processes. 2. Stream allocation and case studies. Ind Eng Chem Res. 1998;37:3408-13.

11. Van der Vorst G, Aelterman W, De Witte B, Van Langenhove H. Assessment of the integral resource consumption of individual chemical production processes in a multipurpose pharmaceutical production plant: a complex task. Ind Eng Chem Res. 2009;48:5344-50.

12. Raymond MJ, Slater CS, Savelski MJ. LCA approach to the analysis of solvent waste issues in the pharmaceutical industry. Green Chem. 2010;12:1826-34.

13. Bieler PS, Fischer U, Hungerbühler K. Modeling the energy consumption of chemical batch plants-top-down approach. Ind Eng Chem Res. 2003;42:6135-44.

14. Bieler PS, Fischer U, Hungerbühler K. Modeling the energy consumption of chemical batch plants - top-down approach. Ind Eng Chem Res. 2004;43:7785-95.

15. Szijjarto A, Papadokonstantakis S, Fischer U, Hungerbühler K. Bottom-up modelling of the steam consumption in multipurpose chemical batch plants focusing on identification of the optimization potential. Ind Eng Chem Res. 2008;47:7323-34.
16. Shamkishore L, Manmadha Reddy K, Pathy A. Energy Conservation in Pharmaceutical Manufacturing. Pharmaceutical Technology Sourcing and Management. 2011;7(11): online publication. http:// www.pharmtech.com/pharmtech/Energy-Conservation-inPharmaceutical-Manufacturin/ArticleStandard/Article/detail/ 747409. Accessed 05 Dec 2012.

17. Liu H. Improving energy efficiency in a pharmaceutical manufacturing environment-analysis of EUI and cooling load. Master Thesis - Massachusetts Institute of Technology. 2009. http:/hdl.handle.net/1721.1/55229. Accessed 05 Dec 2012.

18. Graf C. Energieeffizente Herstellung von Pharmawasser. Pharm Ind. 2010;72:1797-803.

19. Zhi-dong L, Shu-shen Z, Yun Z, Yong Z, Wei L. Evaluation of cleaner production audit in pharmaceutical production industry: case study of the pharmaceutical plant in Dalian, P. R. China. Clean Tech Environ Policy. 2011;13:195-206.

20. Jacka JM, Keller PJ. Business process mapping: improving customer satisfaction. 2nd ed. Hoboken: Willey; 2009.

21. Biegler LT, Grossmann IE, Westerberg AW. Systematic methods of chemical process design. New Jersey: Prentice Hall; 1997.

22. Howat CS. Analysis of plant performance. In: Perry RH, Green DW, editors. Perry's chemical engineers' handbook. 7th ed. New York: McGraw-Hill; 1997.

23. Wernet G, Conradt S, Isenring HP, Jiménez-González C, Hungerbühler $\mathrm{K}$. Life cycle assessment of fine chemical production: a case study of pharmaceutical synthesis. Int J Life Cycle Assess. 2010;15:294-303.

24. Jiménez-González C, Curzons A, Constable D, Cunningham V. Cradle-to-gate life cycle inventory and assessment of pharmaceutical compounds. Int J LCA. 2004;9:114-21.

25. Jiménez-González C, Overcash M. Energy sub-modules applied in life-cycle inventory of processes. Clean Prod Process. 2000;2: $57-66$.

26. Huijbregts M, Rombouts L, Hellweg S, Frischknecht R, Hendriks J, van de Meent $D$, et al. Is cumulative fossil energy demand a useful indicator for the environmental performance of products. Environ Sci Technol. 2006;40:641-8.

27. The Intergovernmental Panel on Climate Change. Climate Change 2007. 2007. http://www.ipcc.ch/ipccreports/ar4-wg1.htm.

28. Verein Deutscher Ingenieure. VDI-Richtline 4600: Cumulative energy demand, terms, definitions, methods of calculation. Düsseldorf: Verein Deutscher Ingenieure; 1997.

29. U.S. Environmental Protection Agency. ENERGY STAR Performance Ratings - Methodology for Incorporating Source Energy Use. U.S. Environmental Protection Agency. March 2011. http://www.energystar.gov/ia/business/evaluate_performance/site source.pdf. Accessed 19 Sept 2012.

30. International Conference on Harmonisation of Technical Requirements for Registration of Pharmaceuticals for Human Use. ICH Harmonised Tripartite Guideline: Quality Risk Management Q9. Step 4. 9 Nov 2005. http://www.ich.org/fileadmin/Public Web_Site/ICH_Products/Guidelines/Quality/Q9/Step4/Q9_ Guideline.pdf

31. International Conference on Harmonisation of Technical Requirements for Registration of Pharmaceuticals for Human Use. ICH Harmonised Tripartite Guideline: Pharmaceutical Quality System Q10. Step 4. 4 Jun 2008

32. Sugiyama H, Sukowski L, Schmidt R. "Japan Quality" in pharmaceutical technical operations. Part I: understanding differences in quality expectations between Western and Japanese markets. Pharm Ind. 2011;73:754-8.

33. Sugiyama H, Sukowski L, Schmidt R. "Japan Quality" in pharmaceutical technical operations. Part II: building a blueprint for better performance in the Japanese market. Pharm Ind. 2011;73:912-8. http://www.ich.org/fileadmin/Public_Web_Site/ICH_Products/ Guidelines/Quality/Q10/Step4/Q10 Guideline.pdf. Accessed 03 Mar 2014 
34. Sugiyama H, Schmidt R. Realizing Continuous Improvement in Pharmaceutical Technical Operations - Business Process Model in Roche's Parenterals Production Kaiseraugst. In: Bogle I D L, Fairweather M, editors. 22nd European Symposium on Computer Aided Process Engineering, Elsevier; 2012. 422-6.
35. Sugiyama H, Schinzel S, Müller G, Schmidt R. Applying process systems engineering for continuous improvement in pharmaceutical production. Proceedings of the 6th International Conference on Process Systems Engineering (PSE ASIA). 2013; 600-5 